\title{
Andreev interferometer with three superconducting electrodes
}

\author{
Artem V. Galaktionov \\ I.E. Tamm Department of Theoretical Physics, P.N. Lebedev Physical Institute, 119991 Moscow, Russia and \\ Laboratory of Cryogenic Nanoelectronics, Nizhny Novgorod State Technical University, 603950 Nizhny Novgorod, Russia \\ Andrei D. Zaikin \\ Institut für Nanotechnologie, Karlsruher Institut für Technologie (KIT), 76021 Karlsruhe, Germany, \\ I.E. Tamm Department of Theoretical Physics, P.N. Lebedev Physical Institute, 119991 Moscow, Russia, and \\ Laboratory of Cryogenic Nanoelectronics, Nizhny Novgorod State Technical University, 603950 Nizhny Novgorod, Russia \\ Leonid S. Kuzmin \\ Chalmers University of Technology, Gothenburg, Sweden and \\ Laboratory of Cryogenic Nanoelectronics, Nizhny Novgorod State Technical University, 603950 Nizhny Novgorod, Russia
}

(Received 27 April 2012; published 20 June 2012)

\begin{abstract}
We develop a quasiclassical theory of Andreev interferometers with three superconducting electrodes. Provided the tunneling interface resistance between one superconducting electrode and the normal metal strongly exceeds two others, significant current sensitivity to the external magnetic flux is observed only at subgap voltages. If all barrier conductances are comparable, multiple Andreev reflection comes into play and substantial current modulation can be achieved in both subgap and overgap voltage regimes. Our analysis reveals a large variety of interesting features, which can be used for performance optimization of Andreev interferometers.
\end{abstract}

DOI: 10.1103/PhysRevB.85.224523

PACS number(s): 74.45.+c, 74.50.+r, 85.25.Am

\section{INTRODUCTION}

Andreev interferometers are often regarded as potential rivals to superconducting quantum interference devices (SQUIDs) for a number of applications with the possibility to achieve higher sensitivity and readout speed. Such applications range from studying of switching dynamics of individual magnetic nanoparticles ${ }^{1}$ to providing readout for superconducting qubits. $^{2}$

Almost two decades ago Petrashov and coworkers performed experimental analysis of magnetoresistance of mesoscopic normal-superconducting (NS) hybrid structures. ${ }^{3}$ This analysis revealed conductance modulation greatly exceeding universal conductance fluctuations. It was perceived that this modulation should be attributed to the effect of the phase difference $\chi$ between superconducting elements of the hybrid structure influencing the process of Andreev reflection (see Refs. 4 and 5 for a review). Since the normal bars in the crosslike diffusive structures studied in experiments ${ }^{3}$ exceeded the superconducting coherence length $\xi_{0}$, the characteristic energy scale for magnetoresistance modulation should be set by the Thouless energy $\epsilon_{\mathrm{Th}}=D / X^{2}$, where $D$ is the diffusion coefficient and $X$ is the bar length in the cross.

In a diffusive NS structure with good transmission of metallic interfaces the magnitude of the magnetoresistance modulation is expected not to exceed few percents. This estimate is directly related to the so-called reentrance effect in the corresponding NS structures reaching the maximum value below $10 \%{ }^{6,7}$ If, however, tunnel barriers are present in the NS system its conductance can change by much higher values. $^{7}$ This is because Andreev conductance of a tunnel barrier at the NS interface is much smaller than its normal state conductance while a diffusive connector has the same low temperature Andreev conductance as its normal one. Hence, one could expect that magnetoresistance modulation could also be much more pronounced in hybrid NS structures, which contain tunnel barriers. This feature was indeed demonstrated experimentally by Pothier et al. ${ }^{8}$ who observed the maximumto-minimum resistance ratio as a function of $\chi$ to be as high as $\sim 5$. Physical insight into the phase dependence of the Andreev conductance in hybrid NS structures is provided by generalization of the Kirchhoff rules worked out by Nazarov ${ }^{9}$ (see also a study of Andreev interferometers with one normal and $N$ superconducting electrodes ${ }^{10}$ ).

In the experiments discussed so far at least one of the external electrodes was in the normal state. Yet another option to fabricate the interference device is to keep all available electrodes superconducting. In this way one would be able to reduce dissipation. Current harmonics of the current would be generated in this case, which are multiples of the Josephson frequency. However, they can be filtered out and the average current can be measured, which should reveal a dependence on the phase difference $\chi$. Experiments with such structures were recently performed by Meschke et al., ${ }^{11}$ and the corresponding theoretical analysis was developed in Ref. 12.

It should be emphasized that the study of the system of several superconducting electrodes coupled by a piece of normal metal has a history of its own. For example, the Josephson triode was tackled in Ref. 13 by means of Aslamazov-Larkin theory combined with the resistively shunted junction (RSJ) model. A similar approach was employed ${ }^{14}$ in order to analyze the behavior of multiterminal Josephson junctions. Interesting features of such structures, such as bistable states and magnetic flux transfer were demonstrated theoretically in the ballistic limit. ${ }^{15}$ One can also mention a modification of Shapiro steps in multiterminal structures. ${ }^{16}$

In this paper we develop a theoretical analysis for a setup of an Andreev interferometer, which is schematically 


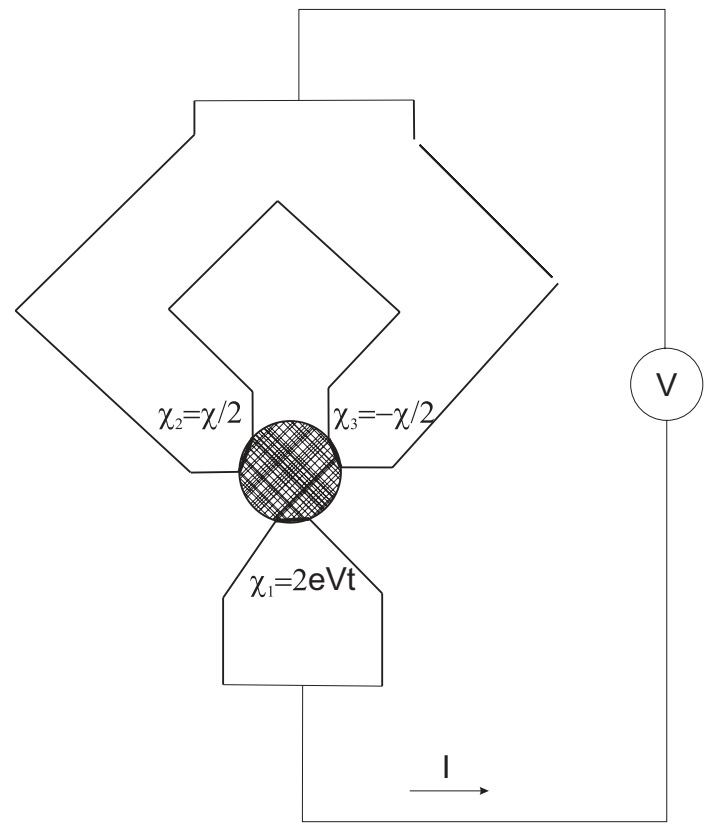

FIG. 1. Scheme of the setup: disordered normal insertion (dot) between three superconducting electrodes. The phase difference $\chi_{2}-$ $\chi_{3}=\chi$ is caused by the magnetic flux piercing the loop.

depicted in Fig. 1. This setup differs from that of Refs. 11 and 12. Our device consists of a disordered normal insertion embedded between three superconducting electrodes. The typical size of this normal insertion $L$ does not exceed the superconducting coherence length $\xi_{0}=\sqrt{D / \Delta}$, where $\Delta$ is the superconducting gap of the electrodes, $D=v_{F} l / 3$, and $l$ is the elastic electron mean free path. At the same time, the normal metal size obeys the condition $L \gg l$. There exists a superconducting phase difference $\chi$ between the electrodes 2 and 3, which is controlled by the external magnetic flux $\Phi$ piercing the superconducting loop (i.e., $\chi=2 \pi \Phi / \Phi_{0}$ ) where $\Phi_{0}$ is the superconducting flux quantum.

In what follows we will generally assume that interfaces between normal metallic dot and superconducting electrodes are weakly transmitting and their normal state conductances $G_{1}, G_{2}$, and $G_{3}$ are supposed to be smaller than the dot conductance $\sim \sigma_{D} \mathcal{A} / L$. Here $\sigma_{D}=2 e^{2} D N_{0}$ is the Drude conductivity of the normal metal, $\mathcal{A}$ is the typical contact area between the normal metal and the electrode, and $N_{0}$ is the density of states at the Fermi surface per spin direction. This relationship between conductances assures the voltage drops only across NS interfaces, while there exist no significant voltage variations inside the normal dot. Under these conditions our results will not depend on the particular shape of the normal metal insertion. For example, one can equally apply our analysis to the setup displayed in Fig. 2. We will also ignore charging effects, which amounts to assuming that all relevant charging energies remain much smaller than the corresponding Josephson coupling energies ${ }^{17}$ and, in addition, that tunneling conductances $G_{1,2,3}$ strongly exceed the quantum conductance unit $G_{q}=e^{2} / h^{18}$. The latter condition also allows us to improve noise characteristics of the systems under consideration.

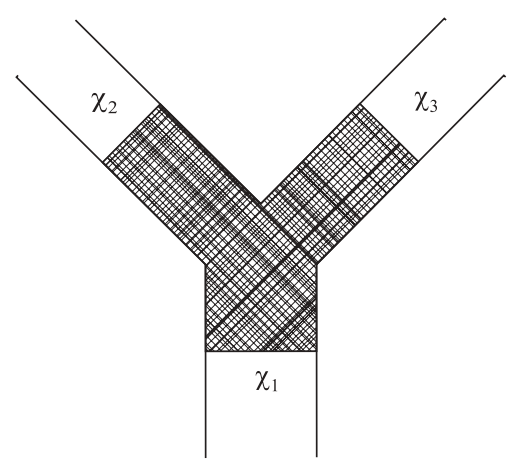

FIG. 2. Another possible realization of interferometer with the Y-shaped normal metal insertion.

In the analysis ${ }^{11,12}$ it was assumed that the contacts between electrodes 2, 3 and the normal insertion are ideal (i.e., highly transmitting) while the first electrode is connected to the dot via a tunnel barrier. Obviously in this case the conductances obey the condition $G_{2} \approx G_{3} \gg G_{1}$. A proximity-induced $\chi$-dependent minigap inside the normal dot develops in this case. As a result, the current-voltage characteristics of such an Andreev interferometer should be sensitive to the phase difference $\chi$, which, in turn, can be controlled by external magnetic flux $\Phi$. Qualitatively the same features hold in the situation studied here as well. On the other hand, there also appear important differences. For instance, we will demonstrate that the properties of the Andreev interferometer essentially depend on the parameter $\gamma \sim \tau_{D} \Delta$, where $\tau_{D}$ is the electron dwell time in the normal dot. Similarly to the case of diffusive superconductor-normal-superconductor (SNS) junctions ${ }^{19}$ this parameter effectively controls the strength of electron-hole dephasing in our device. Another nontrivial feature of our structure is related to the effect of multiple Andreev reflections (MARs) ${ }^{20}$ which becomes particularly important in the case $G_{1} \sim G_{2} \sim G_{3}$ providing significant modifications in the $\chi$-dependent current both in high and low voltage limits. The effect of MARs on the properties of Y-shaped Andreev interferometers was studied in Ref. 21 for a model system with normal insertion substituted by a one-channel quantum wire. Here we will address a realistic configuration, which includes many-channel diffusive conductors of an arbitrary shape.

The structure of our paper is as follows. In Sec. II we outline our general formalism, which allows us to evaluate quasiclassical electron propagators for the problem under consideration. Section III is devoted to the analysis of the density of states in the normal metallic dot as well as the flux-dependent current across our structure in the regime $G_{1} \ll G_{2,3}$. The nonperturbative MAR regime is studied in Sec. IV. We conclude with a summary in Sec. V.

\section{QUASICLASSICAL ANALYSIS}

Our theoretical analysis is performed with the aid of Usadel equations (see, e.g., Ref. 5)

$$
\begin{aligned}
- & D \partial_{\boldsymbol{R}}\left(\check{g} \circ \partial_{\boldsymbol{R}} \check{g}\right)+\check{\tau}_{z} \frac{\partial \check{g}}{\partial t}+\frac{\partial \check{g}}{\partial t^{\prime}} \check{\tau}_{z}+(-i \check{\Delta}(t)+i e \varphi(t)) \check{g} \\
- & \check{g}\left(-i \check{\Delta}\left(t^{\prime}\right)+i e \varphi\left(t^{\prime}\right)\right)=0,
\end{aligned}
$$


where $\check{g}$ represent the quasiclassical propagators that are $4 \times 4$ matrices depending on one spatial and two time variables

$$
\check{g}\left(\boldsymbol{R}, t, t^{\prime}\right)=\left(\begin{array}{cc}
\hat{g}^{R}\left(\boldsymbol{R}, t, t^{\prime}\right) & \hat{g}^{K}\left(\boldsymbol{R}, t, t^{\prime}\right) \\
0 & \hat{g}^{A}\left(\boldsymbol{R}, t, t^{\prime}\right)
\end{array}\right) .
$$

Here $\hat{g}^{R, A, K}$ stand for retarded, advanced, and Keldysh components respectively, each of them forming a $2 \times 2$ matrix. The electric potential is denoted by $\varphi(\boldsymbol{R}, t)$ and $e$ is the electron charge. The product of propagators in Eq. (1) implies time convolution

$$
\left(\check{g}_{1} \circ \check{g}_{2}\right)\left(t, t^{\prime}\right)=\int_{-\infty}^{\infty} d t_{1} \check{g}\left(t, t_{1}\right) \check{g}_{2}\left(t_{1}, t^{\prime}\right) d t_{1} .
$$

The remaining matrices in Eq. (1) are defined as

$$
\check{\tau}_{z}=\left(\begin{array}{cc}
\hat{\tau}_{z} & 0 \\
0 & \hat{\tau}_{z}
\end{array}\right), \quad \hat{\tau}_{z}=\left(\begin{array}{cc}
1 & 0 \\
0 & -1
\end{array}\right), \quad \check{\Delta}=\left(\begin{array}{cc}
\hat{\Delta} & 0 \\
0 & \hat{\Delta}
\end{array}\right),
$$

where

$$
\hat{\Delta}=\left(\begin{array}{cc}
0 & \Delta(\boldsymbol{R}, t) \\
-\Delta^{*}(\boldsymbol{R}, t) & 0
\end{array}\right)
$$

and $\Delta$ is the superconducting order parameter. Quasiclassical propagators (2) also obey the normalization condition

$$
(\check{g} \circ \check{g})\left(t_{1}, t_{2}\right)=\delta\left(t_{1}-t_{2}\right) .
$$

In the immediate vicinity of a tunnel barrier Eqs. (1) are not applicable and the quasiclassical propagators evaluated on both sides of this barrier should be matched by appropriate boundary conditions ${ }^{22}$

$$
2 \sigma_{D} \boldsymbol{n} \check{g} \partial_{\boldsymbol{R}} \check{g}=\sigma_{T}\left[\check{g}_{-}, \check{g}_{+}\right] .
$$

Here the subscripts \pm label the propagators on the right and the left sides of the interface, $\boldsymbol{n}$ is the unit vector perpendicular to the interface (directed from side - to side + ). The combination in the left-hand side of Eq. (5) is continuous at the interface, the commutator in the right-hand side is denoted by brackets, and $\sigma_{T}$ stands for conductivity per unit square of the tunnel barrier.

It is convenient to Fourier transform the quasiclassical propagator with respect to the time difference

$$
\check{g}(\boldsymbol{R}, \epsilon, t)=\int d t^{\prime} \exp \left(i \epsilon t^{\prime}\right) \check{g}\left(\boldsymbol{R}, t+\frac{t^{\prime}}{2}, t-\frac{t^{\prime}}{2}\right) .
$$

Integrating Eq. (1) over the normal metal volume $\mathcal{V}$ and employing the Gauss theorem one finds

$$
\mathcal{V}\left[\epsilon \check{\tau}_{z}, \check{g}\right]=i \int d \mathcal{V} D \partial_{k}\left(\check{g} \partial_{k} \check{g}\right)=i D \oint d S_{k}\left(\check{g} \partial_{k} \check{g}\right) \text {. }
$$

With the aid of the boundary conditions (5) from Eq. (7) we obtain

$$
[\check{Z}, \check{g}]=0, \quad \check{Z}=\sum_{n} \alpha_{n} \check{g}_{n}-i \gamma \frac{\epsilon}{|\Delta|} \check{\tau}_{z},
$$

where the unperturbed quasiclassical propagator of the $n$th electrode is denoted by $\breve{g}_{n}$ and we also introduced the notation

$$
\alpha_{n}=\frac{G_{n}}{G_{1}+G_{2}+G_{3}}, \quad n=1,2,3 .
$$

Finally, we introduce the parameter

$$
\gamma=\frac{2 \sigma_{D} \mathcal{V}|\Delta|}{D\left(G_{1}+G_{2}+G_{3}\right)} \sim \tau_{D}|\Delta|,
$$

which is a direct generalization of the analogous parameter defined for diffusive SNS junctions. ${ }^{19}$ This parameter effectively controls the strength of electron-hole dephasing in our system.

Combining Eq. (8) with the normalization condition (4) we arrive at the expression for the quasiclassical propagator in the normal dot. It reads

$$
\check{\bar{g}}=\frac{\check{Z}}{\sqrt{\check{Z}^{2}}} .
$$

We note that such an expression was previously discussed in Ref. 23 in the context of the full-counting statistics and in Ref. 19 in the context of MAR in SNS junctions.

\section{DENSITY OF STATES AND TUNNELING CURRENT}

Let us first consider the limit $G_{1} \ll G_{2}, G_{3}$. In this case Eq. (8) yields

$$
\check{Z}=\frac{G_{2} \check{g}_{2}+G_{3} \check{g}_{3}}{G_{2}+G_{3}}-i \gamma \frac{\epsilon}{|\Delta|} \check{\tau}_{z} .
$$

The retarded and advanced components of the equilibrium quasiclassical propagators of the electrodes 2 and 3 are

$$
\hat{g}^{R, A}(\epsilon)=\frac{\epsilon \hat{\tau}_{z}+\hat{\Delta}}{\xi^{R, A}}, \quad \xi^{R, A}= \pm \sqrt{(\epsilon \pm i \delta)^{2}-|\Delta|^{2}},
$$

while the Keldysh component is defined as $\hat{g}^{K}=\hat{g}^{R} F-F \hat{g}^{A}$, where $F(\epsilon)=\tanh (\epsilon / 2 T)$ is the Fourier transform of the function $F(t)=-i T / \sinh [\pi T t]$. We also assume that there exists the superconducting phase difference between the order parameters in the electrodes 2 and 3 (i.e., in these two electrodes we define $\Delta=|\Delta| e^{ \pm i \chi / 2}$ ). As we already pointed out, this phase difference is proportional to external magnetic flux $\Phi$ piercing the superconducting ring.

Considering the diagonal component of the matrix $\left(\hat{g}^{R}-\right.$ $\left.\hat{g}^{A}\right) / 2$ and employing Eq. (11) we recover the density of states in the normal dot equal to

$$
n(\epsilon)=\operatorname{Re} \frac{\epsilon}{\sqrt{\epsilon^{2}-\tilde{\Delta}^{2}}},
$$

where we define

$$
\tilde{\Delta}=\frac{\epsilon_{g}}{1+\gamma \sqrt{1-\epsilon^{2} /|\Delta|^{2}}} .
$$

Here the parameter

$$
\epsilon_{g}=|\Delta| \sqrt{1-\frac{4 G_{2} G_{3}}{\left(G_{2}+G_{3}\right)^{2}} \sin ^{2} \frac{\chi}{2}}
$$

represents the minigap in the density of states of the normal dot provided the dephasing parameter tends to zero $\gamma \rightarrow 0$. In the symmetric case $G_{2}=G_{3}$ Eqs. (14)-(16) coincide with the corresponding expressions. ${ }^{24}$

In order to interpret the result (16) in a general case $G_{2} \neq$ $G_{3}$ we recollect that the maximum transmission value $T_{\max }$ for the system of two tunnel barriers with transmissions $T_{2}$ and 

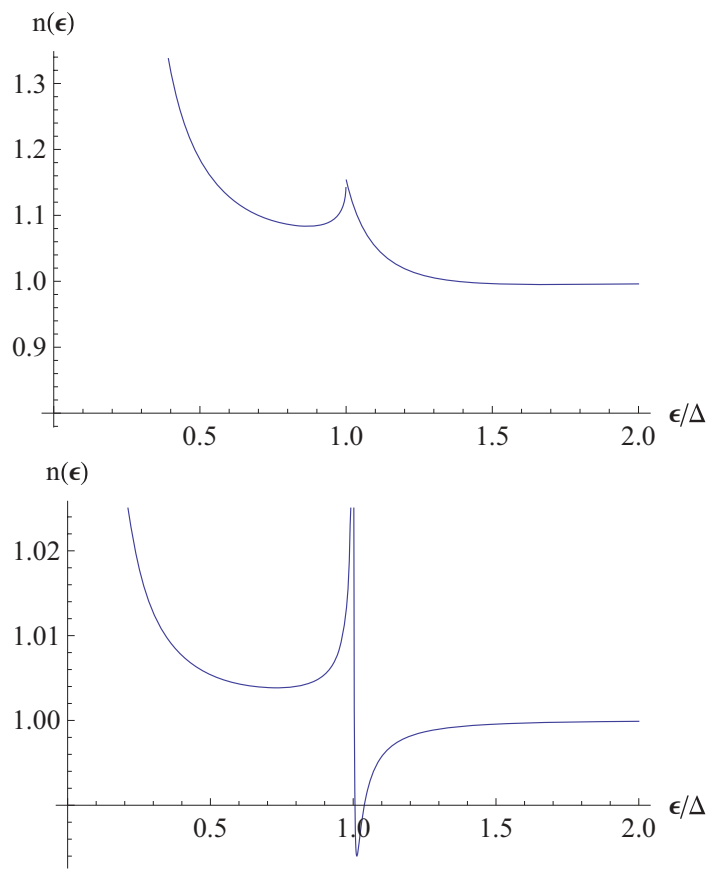

FIG. 3. (Color online) The density of states defined in Eqs. (14)(16) with $\epsilon_{g}=0.5|\Delta|$. The value of $\gamma$ is 1.0 for the upper plot and 10 for the lower plot.

$T_{3}$ (both much smaller than one) is defined by the well known formula

$$
T_{\max }=\frac{4 T_{2} T_{3}}{\left(T_{2}+T_{3}\right)^{2}} .
$$

It follows immediately that Eq. (16) just defines the position of the Andreev level with energy

$$
\epsilon_{A}(\chi)=|\Delta| \sqrt{1-T_{\max } \sin ^{2}(\chi / 2)} .
$$

For nonzero values $\gamma$ the minigap $\Delta_{g}$ in the normal dot is obtained from the solution of the following equation

$$
\Delta_{g}=\frac{\epsilon_{g}}{1+\gamma \sqrt{1-\Delta_{g}^{2} /|\Delta|^{2}}},
$$

(i.e., in the limit of strong dephasing $\gamma \gg 1$ the minigap gets reduced as $\left.\Delta_{g} \approx \epsilon_{g} / \gamma\right)$. The corresponding density of states is exemplified in Fig. 3.

Let us now evaluate the dissipative current flowing across our device at a given voltage bias $V$. In the limit $G_{1} \ll G_{2,3}$ considered in this section the voltage drop concentrates at the tunnel barrier between the first electrode and the normal metallic dot. The current across this barrier reads

$$
\begin{aligned}
I= & \frac{1}{2 e R_{N}} \int_{-\infty}^{\infty} d \epsilon n(\epsilon) n_{0}(\epsilon+e V) \\
& \times\left(\tanh \frac{\epsilon+e V}{2 T}-\tanh \frac{\epsilon}{2 T}\right),
\end{aligned}
$$

where $R_{N} \approx 1 / G_{1}$ and

$$
n_{0}(\epsilon)=\frac{|\epsilon| \theta(|\epsilon|-|\Delta|)}{\sqrt{\epsilon^{2}-|\Delta|^{2}}}
$$

is the standard BCS density of states in the first electrode. $\theta(x)$ denotes the Heaviside step function. Examples of the

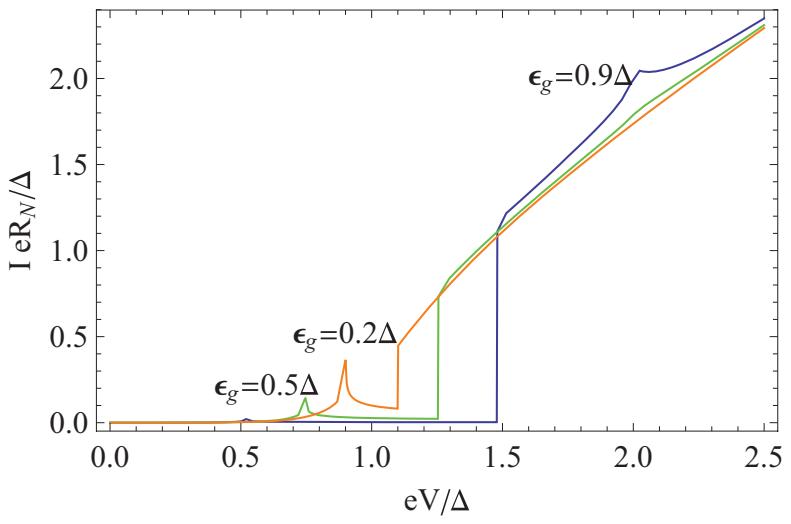

FIG. 4. (Color online) The current-voltage characteristics evaluated from Eq. (18) for $T=0.1 \Delta$ and $\gamma=1$. The values of $\epsilon_{g}$ are $0.9 \Delta, 0.5 \Delta$, and $0.2 \Delta$.

current-voltage characteristics evaluated from Eq. (18) are displayed in Fig. 4. We observe that peculiarities (spikes) on the I-V curve occur at voltages equal to $e V=\Delta-\Delta_{g}$, $e V=\Delta+\Delta_{g}$, and $e V=2 \Delta$ (less pronounced). Here and afterwards $\Delta$ stands for the modulus of the superconducting order parameter.

Typical curves displaying the phase dependence of the tunneling current (18) at voltages $e V>\Delta$ are shown in Fig. 5. We observe that provided temperature is low and $e V<2 \Delta$ there exists a pronounced jump in the current, which occurs as the phase $\chi$ reaches the value corresponding to $e V=\Delta+\Delta_{g}(\chi)$. For $e V>2 \Delta$ the phase dependence of the current is monotonous, the current decreases with growing $\chi$ implying positive magnetoresistance of our structure. The current modulation amplitude decreases rapidly as the voltage increases and becomes almost negligible already at $e V \gtrsim 3 \Delta$.

As long as temperature is not too low and the value $\exp (-\Delta / 2 T)$ is not vanishingly small the $\chi$-dependent current is also observed at voltages $e V<\Delta$. The corresponding plot is shown in Fig. 6. One observes a pronounced current peak which occurs at $e V=\Delta-\Delta_{g}(\chi)$.

In order to complete this part of our analysis we should add that in order to avoid hysteretic phenomena in our Andreev

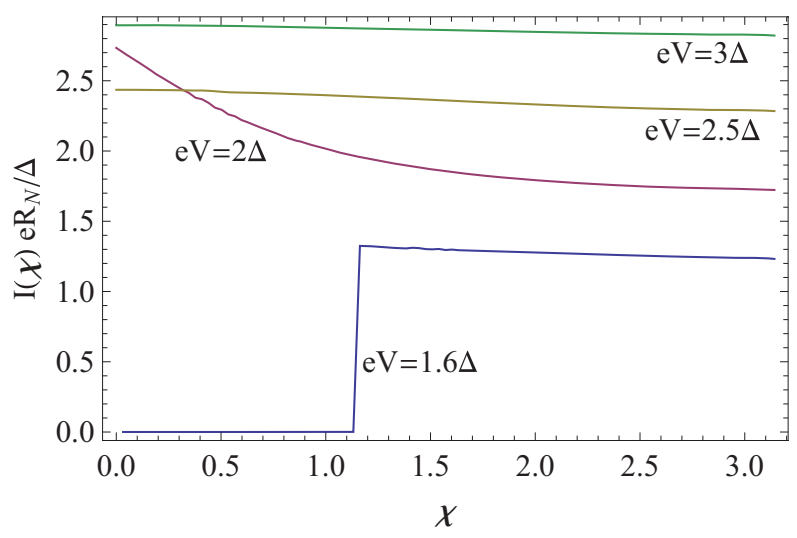

FIG. 5. (Color online) Phase-dependent tunneling current in a symmetric device at $T=0.1 \Delta$ and $\gamma=0.5$. 


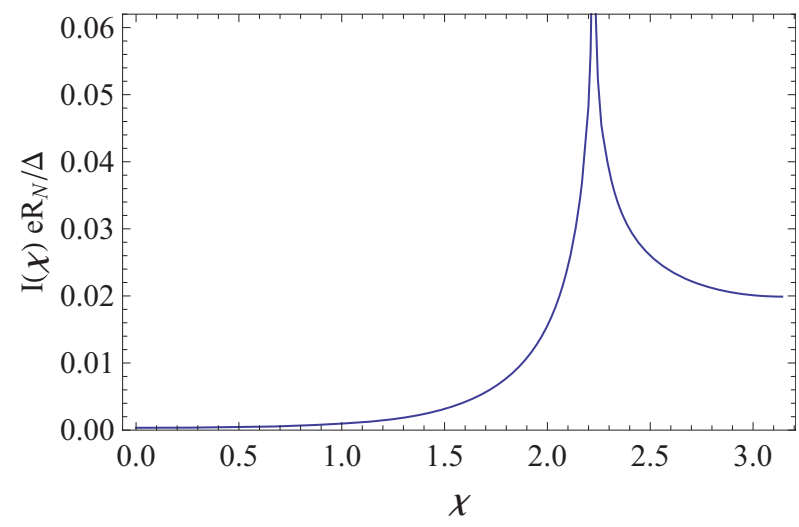

FIG. 6. (Color online) Phase-dependent tunneling current in a symmetric device at $T=0.1 \Delta, \gamma=0.5$, and $e V=0.7 \Delta$.

interferometer it is necessary to obey the condition

$$
\frac{\mathcal{L} I_{C}}{c}<\frac{\Phi_{0}}{2 \pi}
$$

which is exactly analogous to that well known for standard SQUIDs (see, e.g., Ref. 25). Here $\mathcal{L}$ is the loop inductance and $I_{C}$ is the critical Josephson current in our device. The value $I_{C}$ can roughly be estimated with the aid of the AmbegaokarBaratoff formula for the Josephson current

$$
I_{S}(\chi)=\frac{\pi \Delta}{2 e R_{23}} \sin \chi \tanh \frac{\Delta}{2 T}
$$

with $R_{23}=G_{2}^{-1}+G_{3}^{-1}$. Strongest deviations from this formula occur for symmetric structures with $G_{2}=G_{3}$ provided $\gamma \rightarrow 0$. In order to estimate these deviations in this case we can use the following transmission distribution of conducting channels $^{26}$

$$
P\left(T_{n}\right) \propto \frac{1}{T_{n}^{3 / 2} \sqrt{1-T_{n}}} .
$$

Employing this distribution and evaluating the Josephson current, in the limit $T \rightarrow 0$ we obtain

$$
I_{S}(\chi)=\frac{\Delta}{e R_{23}} \sin \chi \mathrm{K}\left(\sin ^{2} \frac{\chi}{2}\right) .
$$

Here $\mathrm{K}(x)=\int_{0}^{\pi / 2} d \phi / \sqrt{1-x \sin ^{2} \phi}$ is the complete elliptic integral. The maximum current value provided by Eq. (23) is higher than that determined from Eq. (21) by a factor $\approx 1.22$ [i.e., maximum deviations from Eq. (21) are in the range of $20 \%]$.

\section{BEYOND TUNNELING LIMIT: MULTIPLE ANDREEV REFLECTION REGIME}

In the previous section we restricted the analysis of I-V curves to the tunneling limit (i.e., evaluated the current in the lowest order in the conductance of the tunnel barrier with lowest transmission $\left.G_{1} \ll G_{2,3}\right)$. If this condition is violated (e.g., all three conductances have approximately the same value $G_{1} \approx G_{2} \approx G_{3}$ ) it is necessary to go beyond the lowestorder perturbation theory and account for higher-order electron tunneling processes between superconducting terminals. In this case an important role is played by the mechanism of multiple Andreev reflection (MAR): quasiparticles with energies below the superconducting gap $\Delta$ propagating inside the normal dot and suffering Andreev reflections at different NS interfaces (i.e., being converted from electrons to holes and back) are accelerated by the electric field and eventually leave the dot area as soon as their energies exceed $\Delta$. Recently it was demonstrated theoretically that this process essentially influences the I-V curves of diffusive SNS junctions. ${ }^{19}$ The system under consideration here is more complicated since it contains three superconducting terminals.

Let us set the electrostatic potential of the first electrode equal to $-V$. Then the quasiclassical electron propagator in this electrode reads

$$
\check{g}_{1}\left(t, t^{\prime}\right)=e^{i e V \check{t}_{z} t} \check{g}_{\mathrm{eq}}\left(t-t^{\prime}\right) e^{-i e V \check{\tau}_{z} t^{\prime}},
$$

where the equilibrium propagator $\check{g}_{\text {eq }}(t)$ is defined in Eq. (13). The general expression for the current flowing into this electrode through the tunneling barrier with conductance $G_{1}$ has the form

$$
I(t)=\frac{\pi}{8 e} G_{1} \operatorname{Tr}\left(\hat{\tau}_{z}\left[\check{\bar{g}}, \check{g}_{1}\right]^{K}\right)(t, t),
$$

where the superscript $K$ denotes the Keldysh component of the commutator. The electron propagator inside the normal dot $\check{g}$ is given by Eq. (11), where the matrix $\check{Z}$ is defined in Eq. (8). One can also cast the expression for $\check{g}$ to the form

$$
\check{\bar{g}}=\frac{1}{\pi} \int_{-\infty}^{\infty} d \lambda \check{K}(\lambda), \quad \check{K}(\lambda)=(\check{Z}+i \lambda)^{-1} .
$$

Provided all electrodes are in the normal state the above equations just yield Kirchhoff rules, i.e.,

$$
I=\frac{G_{1}}{G_{1}+G_{2}+G_{3}}\left[G_{2}\left(V_{2}-V_{1}\right)+G_{3}\left(V_{3}-V_{1}\right)\right] .
$$

In what follows we will make use of this expression in order to normalize the corresponding results derived below for the superconducting case.

It is convenient to rewrite the dependence of the quasiclassical propagators (6) on $\epsilon$ and $t$ with the aid of a series in multiples of the Josephson frequency, i.e.,

$$
\check{A}(\epsilon, t)=\sum_{m=-\infty}^{\infty} \check{A}(\epsilon, m) e^{-2 i m e V t} .
$$

In this representation the matrix $\check{Z}+i \lambda$ has only $m=-1,0,1$ components, which will be denoted as $\breve{H}_{-1}(\epsilon), \breve{H}_{0}(\epsilon, \lambda)$, and $\breve{H}_{1}(\epsilon)$ respectively. The condition

$$
(\check{Z}+i \lambda) \circ \check{K}=\delta\left(t-t^{\prime}\right)
$$

reduces to

$$
\begin{aligned}
& \check{H}_{0}(\epsilon+m e V, \lambda) \check{K}_{\lambda}(\epsilon, m) \\
& \quad+\check{H}_{1}(\epsilon+(m-1) e V) \check{K}_{\lambda}(\epsilon-e V, m-1) \\
& \quad+\check{H}_{-1}(\epsilon+(m+1) e V) \check{K}_{\lambda}(\epsilon+e V, m+1)=\delta_{m, 0} .
\end{aligned}
$$

Introducing $\tilde{K}_{m}(\epsilon, \lambda)=\check{K}_{\lambda}(\epsilon+m e V, m)$, one can rewrite Eq. (29) in the form

$$
\begin{aligned}
& \check{H}_{0}(\epsilon+2 m e V, \lambda) \tilde{K}_{m}(\epsilon, \lambda) \\
& \quad+\check{H}_{1}(\epsilon+(2 m-1) e V) \tilde{K}_{m-1}(\epsilon, \lambda) \\
& \quad+\check{H}_{-1}(\epsilon+(2 m+1) e V) \tilde{K}_{m+1}(\epsilon, \lambda)=\delta_{m, 0} .
\end{aligned}
$$


In order to resolve this equation one can employ the ansatz ${ }^{19}$

$$
\begin{aligned}
& \tilde{K}_{m}=\check{S}_{m} \check{S}_{m-1}, \ldots, \check{S}_{1} \tilde{K}_{0} ; \quad m>0, \\
& \tilde{K}_{m}=\check{P}_{m} \check{P}_{m+1}, \ldots, \check{P}_{-1} \tilde{K}_{0} ; \quad m<0,
\end{aligned}
$$

with the aid of which Eq. (30) yields recurrences relating $\check{S}_{m}$ and $\check{S}_{m+1}$

$$
\begin{aligned}
\check{S}_{m}(\epsilon, \lambda)= & -\left\{\check{H}_{-1}[\epsilon+(2 m+1) e V] \check{S}_{m+1}(\epsilon, \lambda)\right. \\
& \left.+\check{H}_{0}(\epsilon+2 m e V, \lambda)\right\}^{-1} H_{1}[\epsilon+(2 m-1) e V] .
\end{aligned}
$$

Similarly, for $m<0$, we obtain the following relationship between $\check{P}_{m}$ and $\check{P}_{m-1}$

$$
\begin{aligned}
\check{P}_{m}(\epsilon, \lambda)= & -\left\{\check{H}_{1}[\epsilon+(2 m-1) e V] \check{P}_{m-1}(\epsilon, \lambda)\right. \\
& \left.+\check{H}_{0}(\epsilon+2 m e V, \lambda)\right\}^{-1} \check{H}_{-1}[\epsilon+(2 m+1) e V] .
\end{aligned}
$$

At $m=0$ we get from Eq. (30)

$$
\begin{aligned}
\tilde{K}_{0}(\epsilon, \lambda)= & {\left[\check{H}_{1}(\epsilon-e V) \check{P}_{-1}(\epsilon, \lambda)\right.} \\
& \left.+\check{H}_{-1}(\epsilon+e V) \check{S}_{1}(\epsilon, \lambda)+\check{H}_{0}(\epsilon, \lambda)\right]^{-1} .
\end{aligned}
$$

Finally, we impose the boundary conditions

$$
\lim _{m \rightarrow \infty} \check{S}_{m}=0, \quad \lim _{m \rightarrow-\infty} \check{P}_{m}=0 .
$$

Thus, proceeding numerically we set $\check{S}_{m}, \check{P}_{m}=0$ for some large $|m|$ and then employ the recurrences (32) and (33) in order to find $\check{S}_{1}, \check{P}_{-1}$ and $\tilde{K}_{0}$.

Averaging of Eq. (25) results in the following expression for the current:

$$
\begin{aligned}
I= & \int d \lambda \int d \epsilon \operatorname{Tr}\left[\check{S}_{1}(\epsilon, \lambda) \tilde{K}_{0}(\epsilon, \lambda) \check{L}_{-1}(\epsilon+e V)\right. \\
& \left.+\check{P}_{-1}(\epsilon, \lambda) \tilde{K}_{0}(\epsilon, \lambda) \check{L}_{1}(\epsilon-e V)+\tilde{K}_{0}(\epsilon) \check{L}_{0}(\epsilon)\right],
\end{aligned}
$$

which demonstrates that our numerical procedure consists of performing the double integral in $\epsilon, \lambda$ and employing the matrix recurrence relations (32) and (33) at each step of the integration. The matrices depend on dimensionless parameters $\gamma, e V / \Delta, T / \Delta, \chi$, and $\alpha_{2,3}$ (the parameter $\alpha_{1}$ is excluded by $\left.\alpha_{1}=1-\alpha_{2}-\alpha_{3}\right)$.

Let us now present some results of our numerical analysis of the problem in question. ${ }^{27}$ To begin with, it is satisfactory to observe that several important features established in the lowest

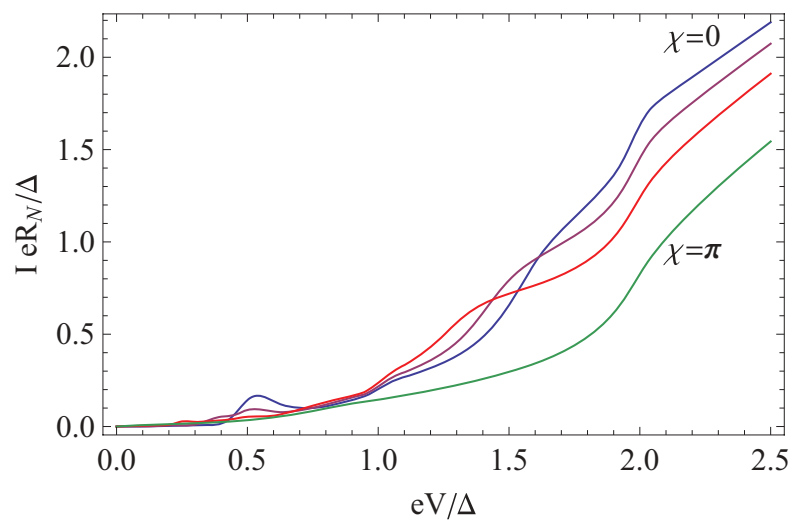

FIG. 7. (Color online) Current-voltage characteristics at $\alpha_{2}=$ $\alpha_{3}=0.45, T=0.1 \Delta$, and $\gamma=1$. The values of $\chi$ are $0,1.3,2$, and $\pi$. The lower $\chi$ value corresponds to the upper curve for $e V>2 \Delta$.

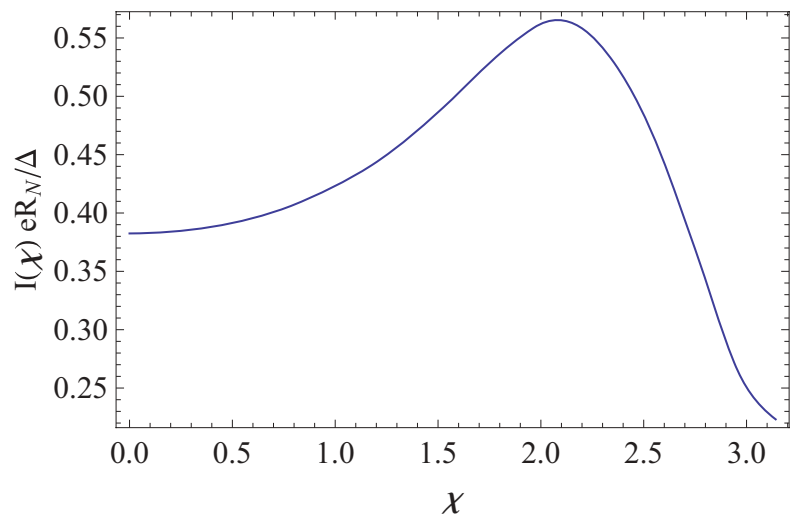

FIG. 8. (Color online) Phase-dependent current at $\alpha_{2}=\alpha_{3}=$ $0.45, T=0.1 \Delta, \gamma=1$, and $e V=1.3 \Delta$.

order in tunneling survive-though possibly with significant quantitative modifications - also within our nonperturbative analysis, which includes the effects of MAR. For example, in Fig. 7 we display the I-V curves evaluated at different values of the phase $\chi$ for the value $G_{1}$ about 4.5 times smaller than $G_{2}$ and $G_{3}$. All our results are normalized to the normal resistance of the structure $R_{N}$ defined from Eq. (27) as

$$
\frac{1}{R_{N}}=\left(\alpha_{2}+\alpha_{3}\right)\left(1-\alpha_{2}-\alpha_{3}\right)\left(G_{1}+G_{2}+G_{3}\right) .
$$

Comparing the results presented in Fig. 7 with those obtained perturbatively (cf., e.g., Fig. 4) in both cases we observe peculiar gaplike features at $e V=\Delta+\Delta_{g}$ in the I-V curves at voltages $\Delta<e V<2 \Delta$. These features are also qualitatively consistent with the results ${ }^{21}$ obtained for a model of Y-shaped Andreev interferometer with a normal part being substituted by a single-mode quantum wire. For instance, this simple model allows us to predict current peaks at voltages $e V=\epsilon_{A}(\chi)$ and $e V=\Delta+\epsilon_{A}(\chi)$, where $e V=\epsilon_{A}(\chi)$ is the phase-dependent energy of the Andreev subgap bound state. These peaks have the same physical origin as those found here at $e V=\Delta_{g}(\chi)$ (cf. the curves displayed in Fig. 7 at $e V<\Delta$ ) and $e V=$ $\Delta+\Delta_{g}(\chi)$, except in our case the current peaks are modified both due to specific transmission distribution in our structure and due to the influence of the parameter $\gamma$.

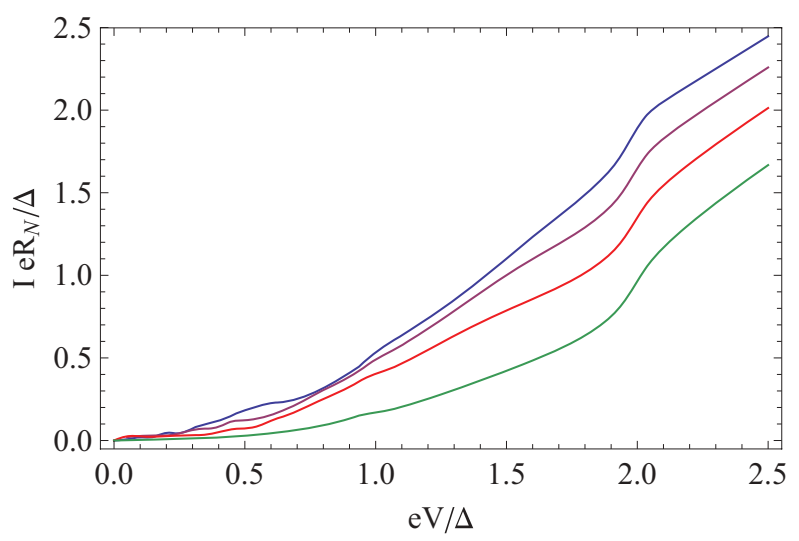

FIG. 9. (Color online) Current-voltage characteristics for $\alpha_{2}=$ $\alpha_{3}=1 / 3, \gamma=1$, and $T=0.1 \Delta$ case. The values of $\chi$ are $0,1.3,2$, and $\pi$ (top to bottom). 


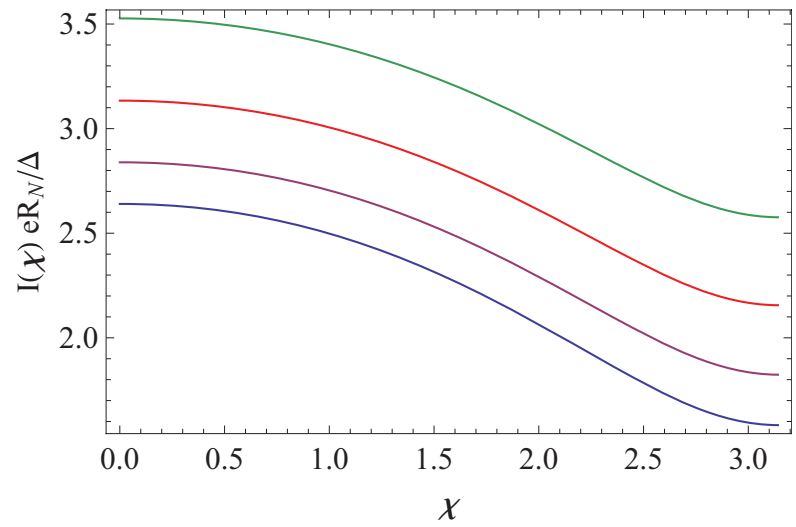

FIG. 10. (Color online) The phase-dependent current in the symmetric case $\left(\alpha_{2}=\alpha_{2}=1 / 3\right)$ for $\gamma=0.5, T=0.1 \Delta$, and $e V=$ $2.3 \Delta, 2.5 \Delta, 2.8 \Delta, 3.2 \Delta$ (bottom to top).

A typical current-phase dependence is depicted in Fig. 8. This curve can be qualitatively compared to one evaluated perturbatively and presented in Fig. 5 at voltages $\Delta<e V<$ $2 \Delta$. Though these curves differ quantitatively, their qualitative behavior remains somewhat similar. Namely, in both cases we observe an increase of the current with $\chi$ at smaller phase values followed by its decrease at larger values of $\chi$.

On the other hand, there also exist significant differences between the results obtained in MAR and tunneling regimes. Perhaps the most essential one is the presence of nonzero subgap current observed within the nonperturbative regime even in the limit of low temperatures. Obviously this feature is lacking within the lowest-order perturbation theory in tunneling. The subgap current is well pronounced at $G_{1} \approx$ $G_{2} \approx G_{3}$, as it is demonstrated in Fig. 9. Similarly to the case of SNS junctions, ${ }^{19}$ there exist somewhat chaotic phase -dependent jumps of current in the subgap voltage region. At $\chi=\pi$ the current is strongly suppressed for subgap voltages $e V<\Delta$.

Our results demonstrate that MAR may essentially influence the phase dependence of the current. In this respect it is instructive to compare Figs. 10 and 5. While in the perturbative tunneling limit the current modulation decreases rapidly with increasing voltage (see Fig. 5), there exists a

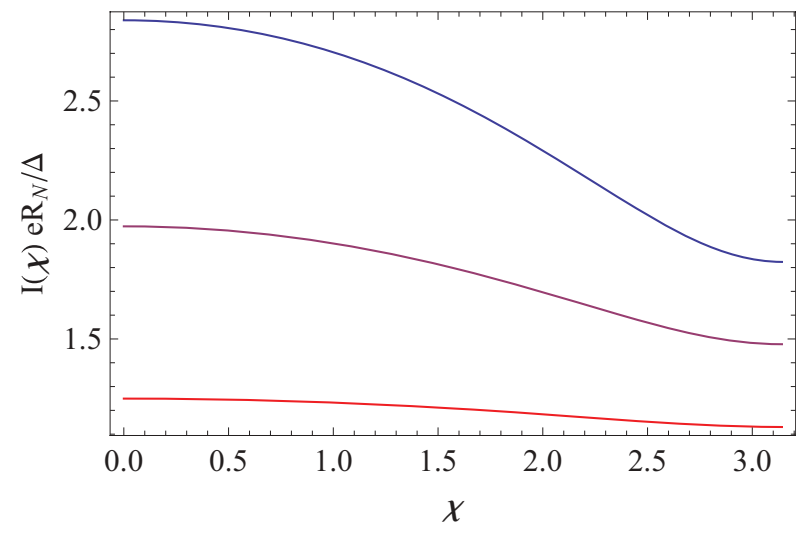

FIG. 11. (Color online) The phase-dependent current in the symmetric case at $e V=2.5 \Delta, T=0.1 \Delta$, and $\gamma=0.5,2,10$ (top to bottom).

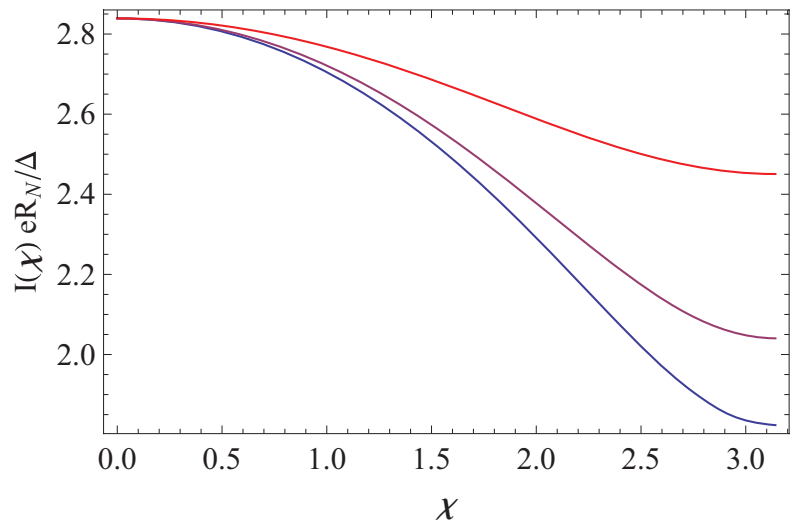

FIG. 12. (Color online) The phase-dependent current at $e V=$ $2.5 \Delta, T=0.1 \Delta$, and $\gamma=0.5$. The lower, middle, and upper curves correspond respectively to $\alpha_{2}=\alpha_{3}=1 / 3$, to $\alpha_{2}=0.444$, $\alpha_{3}=0.222$, and to $\alpha_{2}=0.555, \alpha_{3}=0.111$.

clear voltage-independent modulation of the current in the nonperturbative MAR regime (Fig. 10). This modulation is due to the presence of a phase-dependent excess current with the amplitude $\sim \Delta /\left(e R_{N}\right)$, which is not captured within the lowest-order perturbation theory in tunneling.

Figure 11 illustrates the dependence of the current modulation on the electron-hole dephasing parameter $\gamma$. This modulation clearly decreases with increasing $\gamma$ similarly to the minigap $\Delta_{g}$, as it was discussed in the tunneling limit. It is worth pointing out that conductance asymmetry $G_{2} \neq G_{3}$ also yields a decrease of the current modulation, as it is demonstrated in Fig. 12.

The phase-dependent current at lower voltages is displayed in Fig. 13. Comparing these results to those in Fig. 6 we observe that the current takes much higher values in the nonperturbative MAR regime. At the same time the current-phase dependencies turn out to be considerably smoother in this regime. Note that by tuning the voltage value one can reach the regime where the current depends monotonously on the phase $\chi$ (cf., e.g., the curve evaluated for $e V=0.5 \Delta$ ). In this case the current modulation by the factor $\sim 5$ is observed. This bias voltage regime can be conveniently employed for magnetic flux measurements at lower voltages. This regime appears

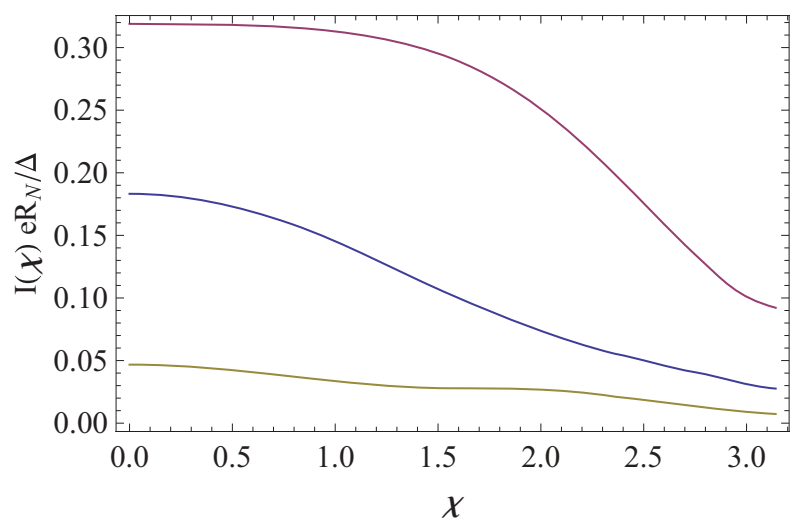

FIG. 13. (Color online) The phase-dependent current of the symmetric junction at $T=0.1 \Delta$ and $\gamma=1$. The voltage values are $0.8 \Delta, 0.5 \Delta$, and $0.2 \Delta$ (top to bottom). 
advantageous as compared to, for example, $e V=0.2 \Delta$, since in the latter case there exist extended flat regions with nearly $\chi$-independent current values.

\section{CONCLUSION}

In this paper we have constructed a general theory of Andreev interferometers with three superconducting electrodes. Our analysis revealed a large variety of different regimes and features, which can be studied experimentally and used for performance optimization of these devices.

In the case $G_{1} \ll G_{2,3}$ the I-V curves can be evaluated perturbatively in the barrier transmissions. In this limiting case an important role is played by the Andreev minigap $\Delta_{g}(\chi)$, which may cause strong dependence of the current on the phase $\chi$ at not very large bias voltages, as shown, for example, in Figs. 5 and 6. On the other hand, current modulation decreases rapidly as $e V$ increases above $2 \Delta$ (see Fig. 5).

If all barrier conductances are comparable, $G_{1} \approx G_{2} \approx G_{3}$, it is necessary to go beyond simple perturbation theory in tunneling and include the effects of MAR into consideration. Features related to the minigap $\Delta_{g}(\chi)$ persist also in this case (cf., e.g., Figs. 4 and 7) but the curves become much smoother and the current signal larger. Significant currentphase modulation can be achieved both in subgap (Fig. 13) and overgap (Figs. 10-12) voltage regimes (i.e., both these regimes can be used for successful operation of Andreev interferometers with three superconducting electrodes). In the regime of large voltages this modulation is due to phasedependent excess current, which is not captured within the perturbative in tunneling analysis.
It is also important to add that both in perturbative and nonperturbative regimes large values of the current modulation can be achieved provided the parameter $\gamma$ defined in Eq. (10) remains sufficiently small. This modulation decreases drastically for large values of $\gamma$ and, hence, such values should be avoided in Andreev interferometers.

Also we would like to make a remark concerning the effect of current noise. Although this effect deserves a separate analysis, it is clear already at this stage that in the MAR regime it would be desirable to avoid working in the limit of low voltages $e V \ll \Delta$, since in this case one could expect dramatic increase of current noise, which could compromise the operation of Andreev interferometers. Theoretical analysis of current noise correlator

$$
\mathcal{S}(\omega)=\int d \tau e^{i \omega \tau}\langle\delta I(t) \delta I(t-\tau)\rangle, \quad \delta I(t)=I(t)-\langle I\rangle
$$

in the case of diffusive SNS junctions in the zero-frequency limit $^{28,29}$ reveals that at low enough voltages one has $\mathcal{S} /\langle I\rangle \propto$ $1 / V$ (i.e., the signal-to-noise ratio should decrease with decreasing voltage). This effect is directly related to MAR. Indeed, an effective charge transferred at voltages $e V \approx 2 \Delta / n$ (with integer $n$ ) equals to $q=n e$. As the number of Andreev reflections $n$ grows with decreasing voltage, the charge $q$ grows as $q \sim(1+2 \Delta /|e V|)$ and, hence, the ratio $\mathcal{S} /\langle I\rangle \propto q$ grows too. We also remark that, as in the case of Andreev interferometers with a normal electrode ${ }^{30}$ one can also expect to observe noise modulation depending on the phase difference $\chi$. However, at this stage we do not expect that this effect could alter our conclusion about the low-voltage regime being possibly problematic for successful operation of Andreev interferometers with three superconducting electrodes.
${ }^{1}$ I. Sosnin, H. Cho, V. T. Petrashov, and A. F. Volkov, Phys. Rev. Lett. 96, 157002 (2006).

${ }^{2}$ V. T. Petrashov, K. G. Chua, K. M. Marshall, R. Sh. Shaikhaidarov, and J. T. Nicholls, Phys. Rev. Lett. 95, 147001 (2005).

${ }^{3}$ V. T. Petrashov, V. N. Antonov, P. Delsing, and R. Claeson, Phys. Rev. Lett. 70, 347 (1993); JETP Lett. 60, 606 (1994); Phys. Rev. Lett. 74, 5268 (1995).

${ }^{4}$ C. J. Lambert and R. Raimondi, J. Phys.: Condens. Matter 10, 901 (1998).

${ }^{5}$ W. Belzig, F. K. Wilhelm, C. Bruder, G. Schön, and A. D. Zaikin, Superlattices Microstruct. 25, 1251 (1999).

${ }^{6}$ Yu.V. Nazarov and T. H. Stoof, Phys. Rev. Lett. 76, 823 (1996).

${ }^{7}$ A. A. Golubov, F. K. Wilhelm, and A. D. Zaikin, Phys. Rev. B 55, 1123 (1997).

${ }^{8}$ H. Pothier, S. Guéron, D. Esteve, and M. H. Devoret, Phys. Rev. Lett. 73, 2488 (1994).

${ }^{9}$ Yu. V. Nazarov, Phys. Rev. Lett. 73, 1420 (1994).

${ }^{10}$ W. Lee, Solid State Commun. 126, 671 (2003).

${ }^{11}$ M. Meschke, J. T. Peltonen, J. P. Pekola, and F. Giazotto, Phys. Rev. B 84, 214514 (2011).

${ }^{12}$ F. Giazotto and F. Taddei, Phys. Rev. B 84, 214502 (2011).

${ }^{13}$ K. K. Likharev, Rev. Mod. Phys. 51, 101 (1979).

${ }^{14}$ R. de Bruyn Ouboter and A. N. Omelyanchouk, Superlattices Microstruct. 25, 1005 (1999).

${ }^{15}$ A. N. Omelyanchouk and M. Zareyan, Physica B 291, 81 (2000).
${ }^{16}$ J. C. Cuevas and H. Pothier, Phys. Rev. B 75, 174513 (2007).

${ }^{17}$ G. Schön and A. D. Zaikin, Phys. Rep. 198, 237 (1990).

${ }^{18}$ A. V. Galaktionov and A. D. Zaikin, Phys. Rev. B 82, 184520 (2010).

${ }^{19}$ E. V. Bezuglyi, E. N. Bratus', and V. S. Shumeiko, Phys. Rev. B 83 , 184517 (2011).

${ }^{20}$ M. Octavio, M. Tinkham, G. E. Blonder, and T. M. Klapwijk, Phys. Rev. B 27, 6739 (1983).

${ }^{21}$ J. Lantz, V. S. Shumeiko, E. Bratus, and G. Wendin, Phys. Rev. B 65, 134523 (2002).

${ }^{22}$ M. Yu. Kupriyanov and V. F. Lukichev, Sov. Phys. JETP 67, 1163 (1988).

${ }^{23}$ J. Börlin, W. Belzig, and C. Bruder, Phys. Rev. Lett. 88, 197001 (2002); P. Samuelsson, Phys. Rev. B 67, 054508 (2003).

${ }^{24}$ E. V. Bezuglyi, V. S. Shumeiko, and G. Wendin, Phys. Rev. B 68, 134506 (2003)

${ }^{25} \mathrm{M}$. Tinkham, Introduction to Superconductivity, 2nd ed. (McGrawHill, New York, 1996).

${ }^{26}$ C. W. J. Beenakker, Rev. Mod. Phys. 69, 731 (1997).

${ }^{27} \mathrm{The} \mathrm{C}++$ code of the numeric programs of this section is available upon request.

${ }^{28}$ D. V. Averin and H. T. Imam, Phys. Rev. Lett. 76, 3814 (1996).

${ }^{29}$ J. C. Cuevas and W. Belzig, Phys. Rev. Lett. 91, 187001 (2003).

${ }^{30}$ B. Reulet, A. A. Kozhevnikov, D. E. Prober, W. Belzig, and Yu. V. Nazarov, Phys. Rev. Lett. 90, 066601 (2003). 